\title{
Effect of Turn-Around Times on the Performance of High Speed Ad-hoc MAC Protocols
}

\author{
Ajay Chandra V. Gummalla and John O. Limb \\ Georgia Insitute of Technology, \\ Atlanta GA 30332, USA
}

\begin{abstract}
Many random access protocols have been proposed for adhoc networks and they are based on collision avoidance principles and are designed for low data rates $(\leq 2 M b p s)$. A key element in the design of high speed MAC protocols is the ability to send feedback from the destination to the source about the state of current transmission. We present an analysis to show that when hardware constraints like transceiver turnaround times are involved, multiplexing the feedback using a different frequency channel is a better design choice. We validate this analysis by comparing the performance of three current ad-hoc MAC protocols as the data rates are increased using a power law model for the variation of the turn-around time. We show that at high data rates, the busy tone protocols will be much more efficient than the collision avoidance protocols. Further, the results show that unless the turnaround times scale in proportion to data rate the performance of CSMA/CA protocols will be worse than slotted-ALOHA
\end{abstract}

\section{Introduction}

Technological advances coupled with the flexibility and mobility of wireless systems are the driving force behind Anyone, Anywhere, Anytime paradigm in wireless networking. Wireless networks that can be rapidly deployed and those that do not need any pre-existing communication infrastructure play an important role in enabling this paradigm. Such network architectures, called ad-hoc networks, are a topic of extensive research. Medium access control (MAC) protocols define rules to allow efficient and fair access to the shared wireless medium and thereby play a crucial rule in determining the performance of such networks. The MAC protocols for such networks should be distributed random access protocols because of their architecture that has no infrastructure.

Wireless random access protocols have been extensively researched and a plethora of protocols proposed. The nature of the wireless channel (time-varying channel; location dependent carrier sensing) and the constraints of the wireless transceivers (half duplex mode of operation; transceiver turnaround times) make the design of ad-hoc wireless MAC protocols very challenging. The scalability of the protocol with data rate adds another dimension to the problem. It is well understood that the design of a MAC protocol to handle these constraints requires 
feedback from the destination node (DN) to the source node (SN) (section 2). There are two options for multiplexing this feedback. One is to use different time slots which we refer to as time duplexed feedback. The other is to use different frequency bands for the data channel and the feedback channel. This is referred to as frequency duplexed feedback. Based on how the feedback is multiplexed all the ad-hoc MAC protocols proposed can be classified into two categories namely; handshaking protocols which use time-duplexed feedback, and busy-tone protocols which use frequency duplexed feedback.

Given the hardware constraints like turn-around times which is the better choice? We present an analysis which shows that frequency duplexed feedback is a better option (section 3). To validate the analysis, we study the performance of the two CSMA/CA based protocols and one busy-tone protocol as data rates are increased, assuming that the turn-around times decrease according to a power law (section 4). We see that as data rates are increased, CSMA/CA based protocols are very inefficient. Further, even at the data rates at which these protocols have been proposed, the efficiency of these protocols is poor when transmitting small packets (section 5).

\section{Motivation}

Carrier sensing wireless protocols have to accommodate many wireless channel characteristics like burst errors, an unreliable channel, location dependent carrier sensing and hardware constraints like turn-around times. The efficiency of a random access protocol is determined by how fast collisions are detected and how soon this information can be conveyed to the source node. In wireline protocols like CSMA/CD, the ability of a node to listen to the medium while transmitting and the fact that a collision on the medium is heard by all nodes listening to the medium results in high performance. In the wireless medium both the above assumptions break down. First, a wireless transceiver cannot be transmitting and listening to the medium at the same time because of self interference (Any transmitted signal that leaks into the receiver usually has a much higher energy than the received signal and hence transceivers cannot listen and transmit at the same time). In the wireless medium the signal strength falls off as a function of distance. Hence, depending on the position of a node relative to the source node, channel sensing will produce different results. This is known as location dependent carrier sensing. Consider the scenario in Fig 1 where $\mathrm{B}$ is in radio coverage of $\mathrm{A}$ and $\mathrm{C}$ and $\mathrm{C}$ is in range of $\mathrm{B}$ and $\mathrm{D}$. If $\mathrm{B}$ is transmitting, $\mathrm{A}$ and $\mathrm{C}$ sense the channel as busy while $\mathrm{D}$ thinks the channel is idle. A transmission from D will corrupt data reception at C. Therefore, location dependent carrier sensing is a serious problem and results in unfair sharing of bandwidth and poor efficiency. Unlike in a wired media, two simultaneous transmissions do not imply a collision. A collision occurs only if the destination node (DN) cannot decode a transmission. Therefore, the DN is the only node that can identify a collision. When a collision is detected, this information should be conveyed to the source node (SN), so that it can abort its transmission and minimize wasted capacity. 

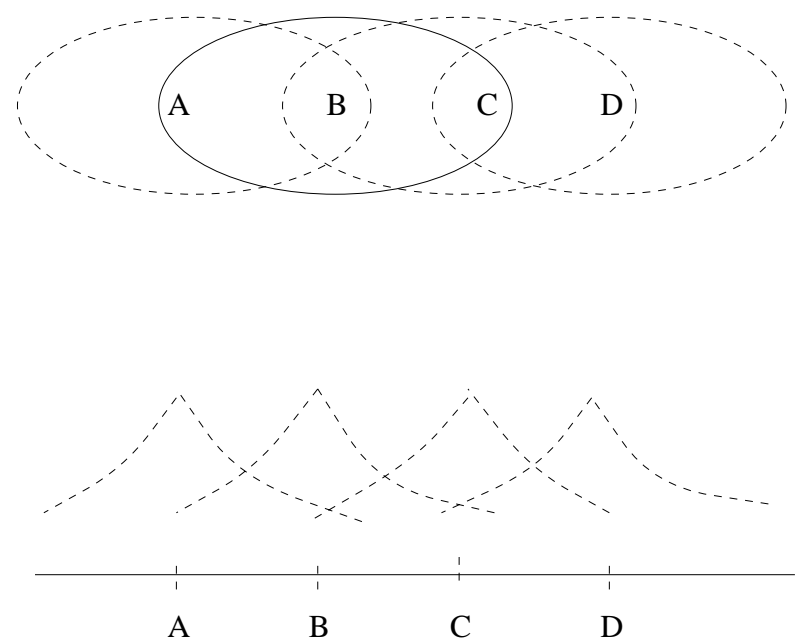

Fig. 1. Logical abstraction of an ad-hoc network.

The feedback problem is: how to enable the destination to convey information about the state of transmission (idle/collision) to the source node? A feedback channel is not available in current wireless systems. Therefore protocols try to minimize collisions by exchanging handshaking messages to reserve the channel for data transmission. This handshaking can be considered as time duplexing of feedback information. Due to the half duplex operation, wireless transceivers would need to switch from receive mode (listening to the channel) to transmit mode (sending data) and vice versa. During such switching there are time periods when the transceiver can neither receive or transmit data. Such switching times also known as turn-around times should be considered in the design of protocols. Consider a turnaround time of $10 \mu \mathrm{s}$. This corresponds to an overhead of 10bits at $1 \mathrm{Mbps}$ and an overhead of 1000bits (125bytes) at 100Mbps. An RTS-CTS-DATA-ACK handshake in the IEEE802.11 MAC protocol requires four turnaround times [6. The second alternative is to use a feedback channel at a different frequency which has a higher hardware cost because of the need for two tranceivers; one each for the data channel and the busy-tone channel. The challenge, then, is: considering the physical channel and hardware limitations which is the most cost-effective method of duplexing the feedback channel?

\section{The Duplexing Problem}

\subsection{Assumptions}

The following assumptions are made about the channel and its capacity. Consider a wireless channel that has bandwidth $B$. This channel is used for communication among multiple users who share the same physical medium. From the channel noise and other constraints, independent of MAC protocol, a maximum data 
rate of $R$ bps can be achieved on this channel. However, when multiple users communicate with each other without any kind of co-ordination in a distributed manner, there is a possibility that multiple users transmit at the same time and such transmissions result in bandwidth being wasted.

Definitions in the analysis (These parameters are illustrated in Fig[2):

Packet Length $=L$ bits

Overhead when a collision occurs $=O_{C}$ bits

Overhead when a successful transmission occurs $=O_{S}$ bits

Overhead when the channel is idle $=O_{I}$ bits

Probability of a successful transmission given a transmission attempt $=P_{S}$

Probability of channel being idle $=P_{I}$

Using the renewal period concept the efficiency is given by [8],

$$
\eta_{m a c}=\frac{P_{S} L}{P_{S}\left(L+O_{S}\right)+O_{I} P_{I}+\left(1-P_{S}\right) O_{C}}
$$

In this equation, the two probabilities $P_{S}$ and $P_{I}$ are a function of the load on the network and the contention resolution algorithms. These probabilities have little effect on the efficiency when the backoff parameters are chosen appropriately. The actual efficiency is controlled by the overheads $\left(O_{C}, O_{I}\right.$ and $\left.O_{S}\right)$ in relation to the packet size. Therefore in this analysis, we take one typical handshaking protocol and one typical frequency-duplexed feedback protocol, estimate the corresponding overheads and determine their approximate efficiency.

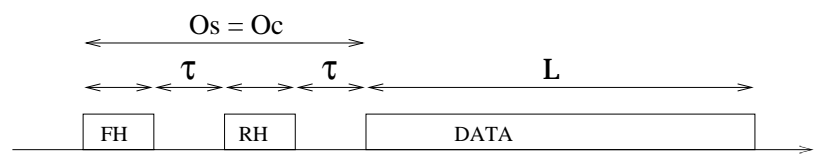

Time Duplex Feedback
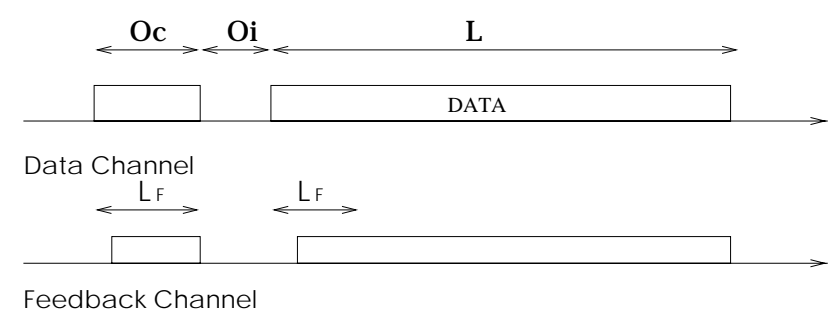

Frequency Duplex Feedback

Fig. 2. Description of different overhead components in the protocols. FH and RH are the forward and reverse handshaking messages. $\tau$ is the turnaround time. 


\subsection{Time Duplexed Feedback Channel (Handshaking Protocols)}

A typical handshaking protocol works as follows: After sensing the channel for a period of time, the source initiates a transmission attempt with a forward handshake $(\mathrm{FH})$. The destination responds with a reverse handshake $(\mathrm{RH})$ if the FH was successful. A successfull FH-RH reserves the channel and data is transmitted. A collision can occur on the $\mathrm{FH}$ or $\mathrm{RH}$ and the resulting overheads are slightly different. However, to understand the influence of these turnaround times let us make the simplifying assumption that the overhead of both types of collision is the same and it is equal to the overhead in a successful transmission. The time spent sensing the channel idle is typically much smaller than the overhead due to collision and successful transmission. Therefore we neglect the overhead of the idle period. Lets consider the overhead in transmitting a single handshake message. A node has to first sense the channel till it finds an idle slot. Once an idle slot is found, the node has to switch from listen mode to transmit mode (1 turnaround period). The node then transmits the handshake packet. This handshake packet has to be large enough such that the destination station can detect the packet transmission $\left(L_{B D}\right.$ symbols), acquire the burst $\left(L_{B A}\right.$ symbols $)$ and then understand the MAC address information $\left(L_{M}\right.$ symbols). Further this packet size has to be greater than a roundtrip delay in the network so that every node can receive this packet unambiguously. Let the number of turnarounds in one successful transmission be $k$ (=3 in Fig. 2) and each turnaround time is $\tau$ secs long. The overhead due to successful transmission or collision is given by,

$$
O=O_{S}=O_{C}=k\left(\tau R+L_{B A}+L_{B D}+L_{M}\right)
$$

and the efficiency is given by,

$$
\eta_{\text {mac }}=\frac{L}{L+\frac{O}{P_{S}}}=\frac{1}{1+k\left(\frac{L_{B A}+L_{B D}+L_{M}+\tau R}{L P_{S}}\right)}
$$

The efficiency of the MAC protocol has an inverse relationship to the data rate if $\tau$ does not scale in proportion to the data rate. Given a fixed turn-around time, the handshaking protocols do not scale well with data rate. Further, each burst transmission has a burst header $\left(L_{B A}+L_{B D}+L_{M}\right)$ which can become a significant overhead when transmitting small packets (e.g. a TCP ACK message is 40 bytes and it comprises $86 \%$ of WWW traffic [9]).

\subsection{Frequency Duplexed Feedback Channel (Busy-Tone Protocols)}

In a frequency duplexed feedback channel a typical packet transaction is as follows: the source after sensing the medium idle will initiate the data transmission. The destination sends the information about success or collision back to the source on the feedback channel. The source node does not know the result of its current transmission till it gets this feedback. The available bandwidth has to be allocated between the data channel and the feedback channel. If we allocate this bandwidth in ratio $\alpha$, the data and feedback channel capacities are related 
as $B_{F}=\alpha B_{D}$ and $R_{F}=\alpha R_{D}$. We can assume that it takes the same time to detect the transmission, acquire the burst and detect the source and destination of the transmission, as in a handshaking protocol. The time to get the feedback to the source is therefore, $\frac{L_{B A}+L_{B D}+L_{M}}{R_{D}}+\tau_{a}$ where $\tau_{a}$ is the time required to assert and detect the feedback tone. The time required to detect the feedback tone should equal $\frac{L_{B D}}{R_{F}}$ (the same time as needed to detect the data carrier, but scaled by the feedback channel bandwidth). Therefore, the overhead in bits is $O=L_{M}+L_{B S}+L_{B A} \frac{1+\alpha}{\alpha}$. The efficiency is given by

$$
\eta_{m a c}=\left(\frac{1}{1+\alpha}\right)\left(\frac{L}{L+\frac{O}{P_{S}}}\right)=\left(\frac{1}{1+\alpha}\right)\left(\frac{1}{1+\frac{L_{M}+L_{B S}+L_{B A} \frac{1+\alpha}{\alpha}}{L P_{S}}}\right)
$$

It can be seen that the efficiency of frequency duplex solutions is independent of the data rate. Also the efficiency is a function of the ratio parameter $\alpha$. For small values of $\alpha$ it takes too long to receive the feedback information resulting in poor efficiency. At the other extreme, if one assigns too much bandwidth to the feedback channel, the time to transmit data increases, thereby decreasing the efficiency. Therefore there is an optimal choice for $\alpha$. The efficiency is maximized when $\alpha=\left(\frac{L_{B D}}{L_{B D}+L_{B A}+L_{M}+L P_{S}}\right)^{\frac{1}{2}}$.

In this analysis it has been shown that handshaking protocols do not scale well to high data rates. The main reason is the turnaround time which does not decrease in proportion to increasing data rates. On the contrary busy tone protocols with frequency duplexed feedback scale well with data rate. The next section studies the performance of two handshaking protocols and one busy tone protocol to justify the above arguments.

\section{Performance of Current Ad-hoc MAC Protocols}

\subsection{Distributed Foundation Wireless MAC (DFWMAC)}

DFWMAC is the basic access protocol in the recently standardized IEEE 802.11 wireless LAN standard [6]. It uses four way handshaking to handle the hidden nodes (RTS-CTS) and an unreliable wireless channel (ACK). When a node has data to transmit, it tries to acquire the channel by sending an RTS packet. If the RTS transmission is received without any errors, the destination node responds with a CTS packet indicating that it is ready to receive the data. The node then completes the packet transmission. If the RTS transmission results in a collision, no CTS is received. When RTS fails or the channel is sensed busy, the node backs off a random amount of time. An analytical expression was derived for the maximum throughput achieved by this protocol when the network is fully loaded [5]. It is,

$$
\eta=\frac{P_{s} E(P)}{E(I)+P_{s} T_{s}+\left(1-P_{s}\right) T_{c}}
$$

where $P_{s}$ is the probability of a successful transmission, $\mathrm{E}(\mathrm{P})$ is the mean packet length, E(I) is the mean idle period duration and $T_{c} \& T_{s}$ the durations of a collision and successful transmission respectively. We use this throughput equation 
derived and verified in [5] with the parameters for the packet sizes and interframe spaces as specified in the IEEE 802.11 standard and vary the data rate from the specified $1 \mathrm{Mbps}$ to $100 \mathrm{Mbps}$. The interframe spaces in this protocol have turnaround times included in them.

\subsection{Elimination Yield-Non Preemptive Multiple Access (EY-NPMA)}

EY-NPMA is the channel access protocol used in the HIPERLAN system developed in Europe [7. The channel access has three phases: prioritization phase (the highest priority is decided), contention phase (nodes of the same priority contend and one station wins) and transmission phase (successful station completes the data transmission) [7. To understand the performance of this protocol as a function of data rate it is necessary to identify parameters in the protocol that scale with data-rate and those that are hardware limitations. Each component of the protocol is examined and their dependence on data rate is listed below.

- Low Bit Rate header: At the beginning of every data packet transmission the standard specifies a portion of the header that should be transmitted at a lower data rate so that most of the circuits need not be turned ON unless necessary. It is possible that if the MAC is upgraded to a higher data rate, the LBR header also might be proportionally increased. In this analysis however, we assume that this is not changed. $\left(T_{L B R}=34 \mathrm{LBR}\right.$ bits $\left.=24 \mu \mathrm{s}\right)$.

- Prioritization Slot $\left(i_{P S}\right)$ and Priority Assertion Slot $\left(i_{P A}\right)$ have a turnaround time embedded in them. A node has to sense the channel if a higher priority node is transmitting (receive mode) and if the channel is idle, the node has to turnaround and assert priority. Hence these slot sizes are hardware dependent. $\left(i_{P A}=i_{P S}=168 \mathrm{HBR}\right.$ periods $\left.=7.14 \mu \mathrm{s}\right)$.

- Elimination phase slot $\left(i_{E S}\right)$ and Survival Verification interval $\left(i_{E S V}\right)$ also have a turnaround time embedded in them. In the elimination phase a sender transmits for a random duration of time and then has to turn around and listen for a survival verification period. Hence these slots have to be longer than one turnaround time and a sensing interval, both of which are dependent on the hardware. $\left(i_{E S V}=i_{E S}=212 \mathrm{HBR}\right.$ periods $\left.=9 \mu \mathrm{s}\right)$.

- The Yield phase slot is similar to prioritization slot $\left(i_{Y S}\right)$ and is of the same size. $\left(i_{Y S}=i_{P S}\right)$.

- Data is transmitted at a higher data rate and the transmission time correspondingly decreases.

- The acknowledgment transmission time decreases with higher data rate. At the end of the data transmission the receiving node has to turn around and send the acknowledgment. $\left(T_{A K}=512 \mathrm{HBR}\right.$ periods $\left.+21 \mu \mathrm{s}\right)$.

We can use the throughput equation derived in [4] with the parameters specified in the standard. The throughput as a function of the data rate is given by,

$$
\eta=\frac{E(P) P(T=1)}{\frac{E(P) 496}{416}+R_{D}\left(i_{P A}+i_{E S V}+i_{A K}+i_{H}\right)+T_{L B R} R_{L}+R_{D}\left(i_{E S} n_{E}+i_{Y S} n_{Y}\right)}
$$


where $\mathrm{E}(\mathrm{P})$ is the mean packet size, $\mathrm{P}(\mathrm{T}=1)$ is the probability that a single transmission occurs during data phase, the ratio $\frac{496}{416}$ is due the error correcting codes specified in the standard, $i_{H}$ is the synchronization interval in the packet header and $n_{E}$ and $n_{Y}$ are the mean lengths of the elimination and yield phases.

\subsection{Receiver Initiated - Busy Tone Multiple Access}

Receiver Initiated - Busy Tone Multiple Access (RI-BTMA), though initially proposed as a modification to BTMA to improve efficiency, was probably the first protocol that took advantage of the fact that the DN is the only node that can tell whether the current transmission is a collision or not 3 . When a node has data to transmit, it samples the busy tone channel. If the busy tone channel is idle, it initiates a data transmission. If this transmission is received at the destination without any errors, the destination station asserts on the busy tone channel. If at the end of the slot, the busy tone channel is not asserted, the transmission is aborted and rescheduled after a random period. An equation was derived for the throughput for this protocol in 3 . It is given by,

$$
\eta=\frac{P_{s}(g+1)}{P_{s}+g}
$$

where, $P_{s}$ is the probability of exactly one packet arrival in a slot and $\frac{1}{g}$ is the mean packet size in slots. The slot is chosen such that it is long enough to detect a collision and transmit the feedback $\left(=\left(L_{B A}+L_{B D}\left(1+\frac{1}{\alpha}\right)+L_{M}\right)\right.$ bits $)$.

\section{Performance Results}

To study the performance of these three protocols as function of data rate we need a model of how the turn-around time changes with data rate. Assuming that the turnaround time will not vary with data rate is very simplistic and probably not very realistic. At the other extreme, the turnaround time does not decrease in proportion to the increase in data rate. In this analysis, we assume a power law variation with rate. $\tau=\frac{\tau_{1}}{R^{(1-\gamma)}}$ where $\tau_{1}$ is the turnaround time at $1 \mathrm{Mbps}$, and $\mathrm{R}$ is the rate of data transmission. $\gamma$ indicates how well $\tau$ scales with data rate and is in the range $[0,1] \cdot \gamma=0$ corresponds to the case where turnaround times scale down in proportion to increase in data rate and $\gamma=1$ corresponds to the case when turnaround times are independent of rate.

The efficiency of DFWMAC and EY-NPMA protocols as a function of data rate and packet size with $\gamma=1$ is shown in Fig. 3 and Fig. 4. The data rate is varied from $1 \mathrm{Mbps}$ to $100 \mathrm{Mbps}$. Three different packet sizes were considered. 8184 bits is the maximum packet size specified in the 802.11 standard and 19080 bits is the maximum message size in the HIPERLAN standard. A third packet size of 1000 bits is considered because it more accurately represents the mean packet size in a typical LAN. It can be seen that the performance of both the ad-hoc wireless MAC protocols decreases monotonically as the data rate is increased. With 1000 bit packets the performance is much worse. At small packet 


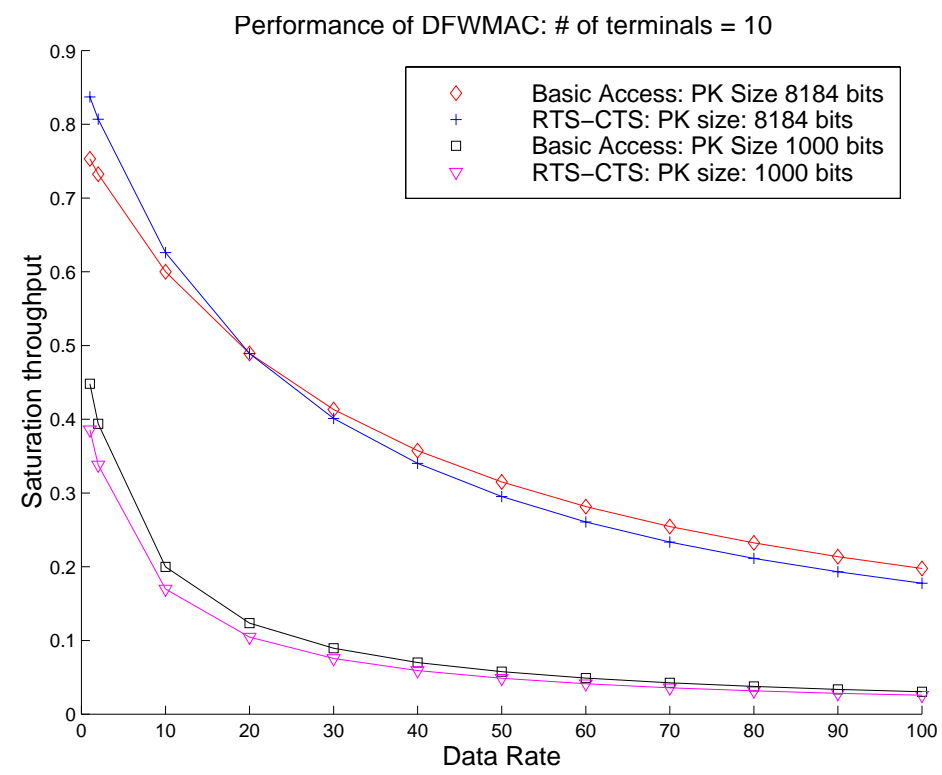

Fig. 3. Performance of DFWMAC as the data rate is increased.

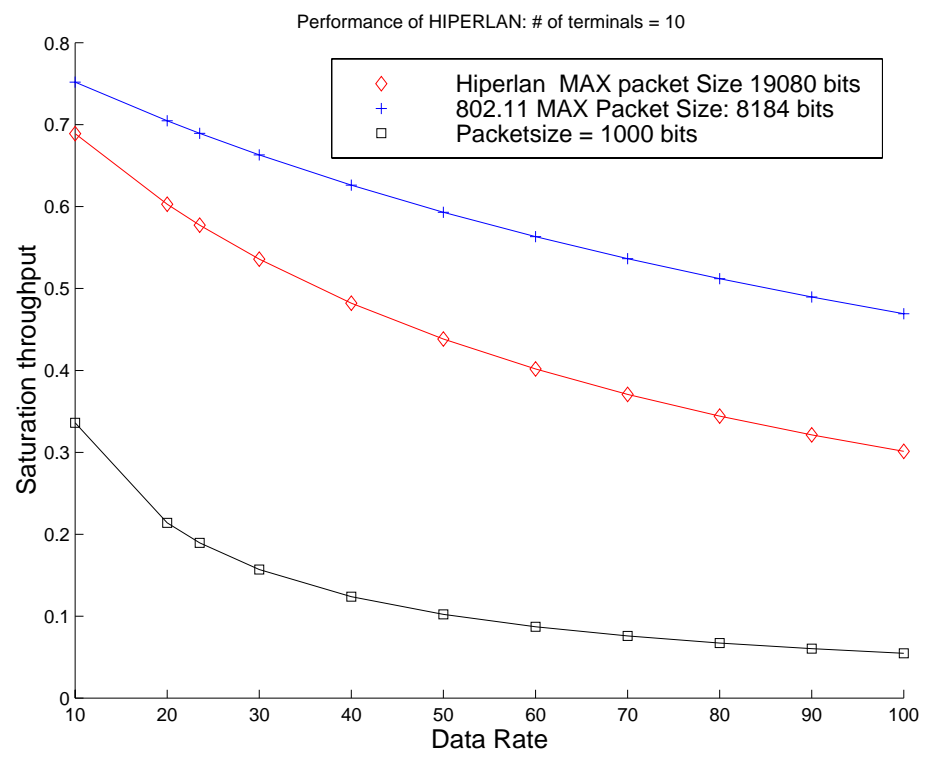

Fig. 4. Performance of EY-NPMA as the data rate is increased. 


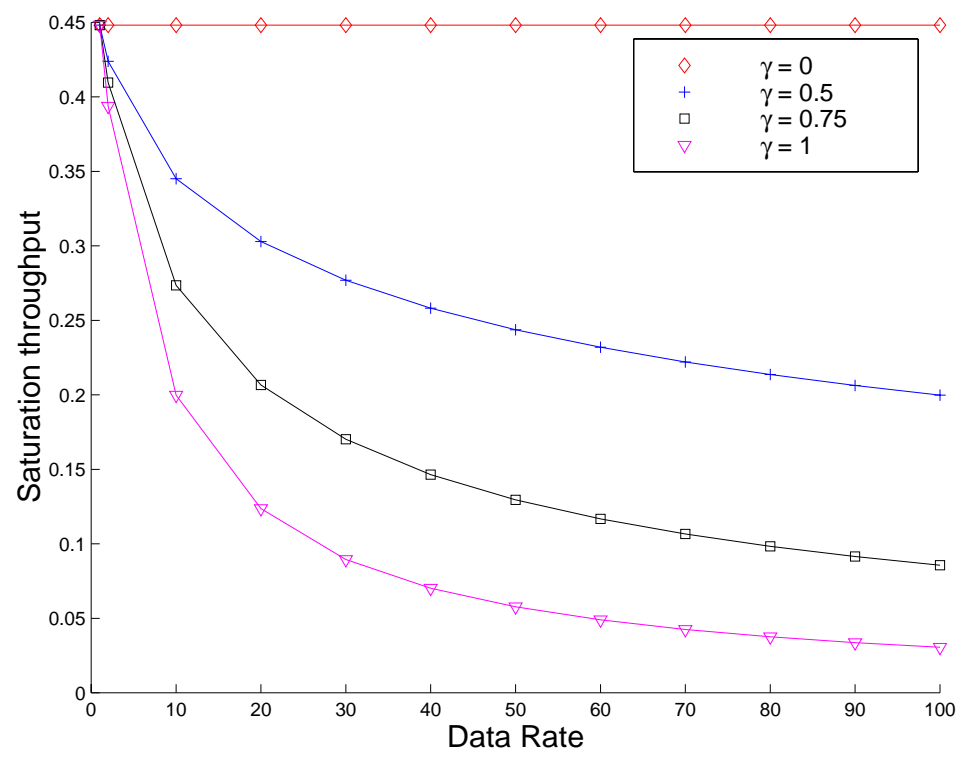

Fig. 5. Performance of DFWMAC for four different values of $\gamma(0.0,0.5,0.75,1.0)$.
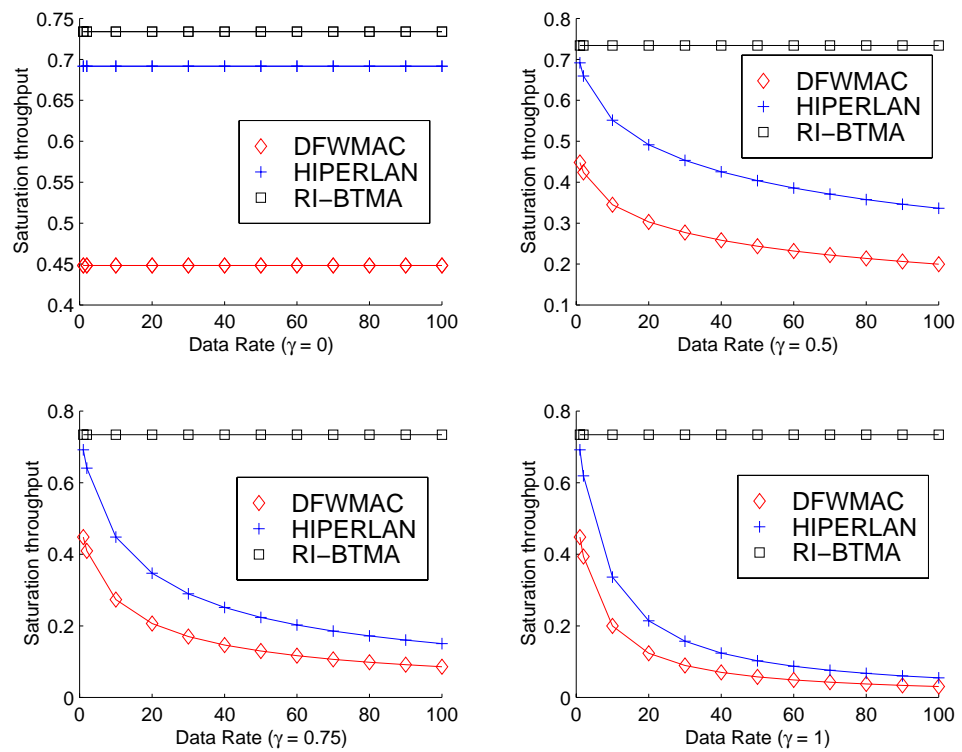

Fig. 6. Performance of DFWMAC, EY-NPMA and RI-BTMA for four different values of $\gamma(0.0,0.5,0.75,1.0)$. 
sizes the efficiency is not better than the simple Slotted-ALOHA even at the data rates at which the standards are proposed. Fig. 5 shows the performance of DFWMAC for 1000 bit messages for different values of $\gamma$. This indicates that the degradation in performance is significant even if the turnaround times decreased by an order of magnitude for two orders of magnitude increase in data rate $(\gamma=0.5)$. Fig 6 compares the three protocols for a 1000 bit packet and different values of $\gamma$. This shows that RI-BTMA protocol's performance is not affected by variations in turn-around times and hence is rate scalable.

\section{Conclusions}

The method used to duplex the feedback information will have a significant impact on the performance of wireless ad-hoc protocols at high data rates. A mathematical analysis was presented to show that when turnaround switching times are involved, busy tone protocols scale well with data rate. The performance of wireless random access protocols proposed in current wireless LAN standards has been studied. We have identified parameters in these MAC protocols that have turnaround periods and hence are critical in the design of high speed MAC protocols. It was shown that as we move to higher data rates these CSMA/CA protocols perform very poorly. This performance loss can be largely attributed to the turnaround times involved in all time-duplex protocols. The results indicate that unless the turnaround times scale in proportion to data rate the performance of CSMA/CA protocols will be worse than S-ALOHA.

\section{References}

1. P.Karn: MACA - A New Channel Access Method for Packet Radio, ARRL/CRRL Amatuer Radio 9th Computer networking Conference, Sept 22,1990.

2. F.A.Tobagi and L. Kleinrock: Packet Switching in Radio Channels: Part II - The Hidden Terminal Problem in Carrier Sense Multiple Access and the Busy Tone Solution, IEEE Trans. Commun., COM-23, 1975 pp 1417-1433.

3. Cheng-Shong Wu and Victor O.K. Li: Receiver-Initiated Busy Tone Multiple Access in Packet Radio Networks, Proc Sigcomm ' 88 pp 336-342.

4. G. Anastasi, L. Lenzini, and E. Mingozzi: Stability and Performance Analysis of HIPERLAN Proc. IEEE INFOCOM'98.

5. Giuseppe Bianchi: IEEE 802.11 Saturation Throughput Analysis, IEEE Commun. Letters, Vol. 2 N0. 12, December 1998.

6. Brian P. Crow, Indra Widjaja, Jeong Geun Kim, and Prescott T. Sakai: IEEE 802.11: Wireless Local Area Networks, IEEE commun. mag. Sept 1997.

7. ETSI: HIPERLAN Functional Specification, ETSI draft standard, July 1995.

8. Raphael Rom and Moshe Sidi: Multiple Access Protocols Performance and Analysis, Springer-Verlag 1990.

9. Hyoung-Kee Choi and John O. Limb: Discussions on the traces collected on Gatech LAN network 